\title{
Aligning House Staff Performance Improvement Projects With Hospital Priorities: An Innovative Approach
}

\author{
Kraftin E. Schreyer, MD, ${ }^{1}$ Susan Coull, MBA, ${ }^{2}$ Henry A. Pitt, MD, ${ }^{3}$ Susan L. Freeman, MD, MS ${ }^{4}$ \\ ${ }^{1}$ Department of Emergency Medicine, Temple University Hospital and Temple University Hospital-Episcopal Campus, Philadelphia, PA \\ ${ }^{2}$ Department of Graduate Medical Education, Lewis Katz School of Medicine at Temple University, and Associate Hospital Director \\ for Medical Education, Temple University Hospital, Philadelphia, PA ${ }^{3}$ Chief Quality Officer, Temple University Health System, \\ Philadelphia, PA ${ }^{4}$ Chief Medical Officer, Temple University Health System, and Vice Dean, Health Care Systems, Lewis Katz School \\ of Medicine at Temple University, Philadelphia, PA
}

Background: As hospital leaders work to meet national performance improvement (PI) priorities and provide high-value healthcare, aligning house staff goals with those of the hospital organization becomes necessary. A hospital leadership goal is to achieve the Institute for Healthcare Improvement (IHI) Triple Aim with the delivery and measurement of high-value care through various PI frameworks, including the domains of the University HealthSystem Consortium (UHC), now Vizient. However, most house staff develop PI projects within their departments, and these projects do not always align with hospital priorities. We sought to determine the extent of alignment between the house staff and the hospital.

Methods: An inventory of house staff projects determined by survey responses from house staff and lists provided by program directors and chairs was compiled during a 2-year period. A team of quality experts mapped all house staff projects to the UHC domains of care and to the IHI Triple Aim and then performed a gap analysis.

Results: A total of 184 projects representing 24 departments were identified. Most projects (38\%), representing 18 departments, were categorized in the UHC Safety domain. The remaining projects were categorized into the domains of Efficiency (23\%), Patient Centeredness (12\%), Effectiveness (12\%), Equity (8\%), and Mortality (7\%). Many departments did not have projects in all domains.

Conclusion: We created unique and concise graphic representations of individual house staff projects aligning with hospital initiatives. Our framework generated an action plan for a proactive approach for continually aligning future house staff PI projects with the hospital goals and national healthcare agendas.

Keywords: Health care quality, health care quality assessment, house staff, quality improvement

Address correspondence to Kraftin E. Schreyer, MD, Department of Emergency Medicine, Temple University Hospital and Temple University Hospital-Episcopal Campus, 1316 W. Ontario Street, 1004 Jones Hall, Philadelphia, PA 19140. Tel: (215) 606-7666.

Email: kraftin.schreyer@tuhs.temple.edu

\section{INTRODUCTION}

A framework for aligning the performance improvement (PI) goals of house staff with those of the hospital organization has not been previously described, despite descriptions of high-functioning house staff quality councils (HSQCs). ${ }^{1,2}$ However, as hospital leaders work to meet national PI priorities and provide high-value healthcare, aligning the goals of house staff with those of hospital administration and medical staff leadership becomes necessary.,4

In the setting of an aging population with chronic health problems, new demands have been placed on healthcare systems. In response, hospital organizations are focused on national agendas described by the elements of the Triple Aim defined by the Institute for Healthcare Improvement
(IHI). ${ }^{5}$ The goals of the IHI Triple Aim are to improve population health and the patient care experience while reducing the per capita cost of healthcare (Figure 1). By providing the highest quality care at the lowest cost, hospitals provide high-value care.

To achieve the goals of the IHI Triple Aim, our hospital has organized an approach around the 6 University HealthSystem Consortium (UHC, now Vizient) domains of care, adopted from the Institute of Medicine: Safety, Mortality, Effectiveness, Efficiency, Patient Centeredness, and Equity (Table). ${ }^{3}$ The domains of care serve as benchmarks for comparison with other academic medical centers. Each domain focuses on a different aspect of patient care: reducing complications of care, reducing inpatient mortality, reducing 
Table. University HealthSystem Consortium Domains of Care

\begin{tabular}{ll}
\hline Domain & \multicolumn{1}{c}{ Description } \\
\hline $\begin{array}{l}\text { Safety } \\
\text { Effectiveness }\end{array}$ & $\begin{array}{l}\text { Reduce complications of care } \\
\text { Reduce inpatient deaths } \\
\text { Reduce inpatient readmission by } \\
\text { focusing on frequently readmitted } \\
\text { patients }\end{array}$ \\
Efficiency & $\begin{array}{c}\text { Reduce inpatient length of stay and cost } \\
\text { per case } \\
\text { Improve patient satisfaction } \\
\text { Patient }\end{array}$ \\
Equity & $\begin{array}{l}\text { Achieve desired healthy community } \\
\text { outcomes through a sustainable, } \\
\text { coordinated model of healthcare } \\
\text { delivery }\end{array}$ \\
\hline
\end{tabular}

readmission rates, reducing inpatient length of stay and cost of care, improving patient satisfaction, and coordinating care to promote a healthy community. Each medical center is compared to others based on calculated absolute to expected ratios of benchmarks within each domain and then ranked within a quartile system. Multidisciplinary teams organized around each UHC domain set goals and create action plans for achieving those goals.

Hospital graduate medical education (GME) committees are simultaneously striving to achieve the goals of the Clinical Learning Environment Review (CLER) that include engaging residents in $\mathrm{PI}$ and using data to improve healthcare systems, reduce healthcare disparities, and improve patient outcomes.

On a smaller but equally important scale, house staff are focused on quality projects within their respective departments. Our institution has an HSQC, a multidisciplinary, resident-led committee consisting of resident members

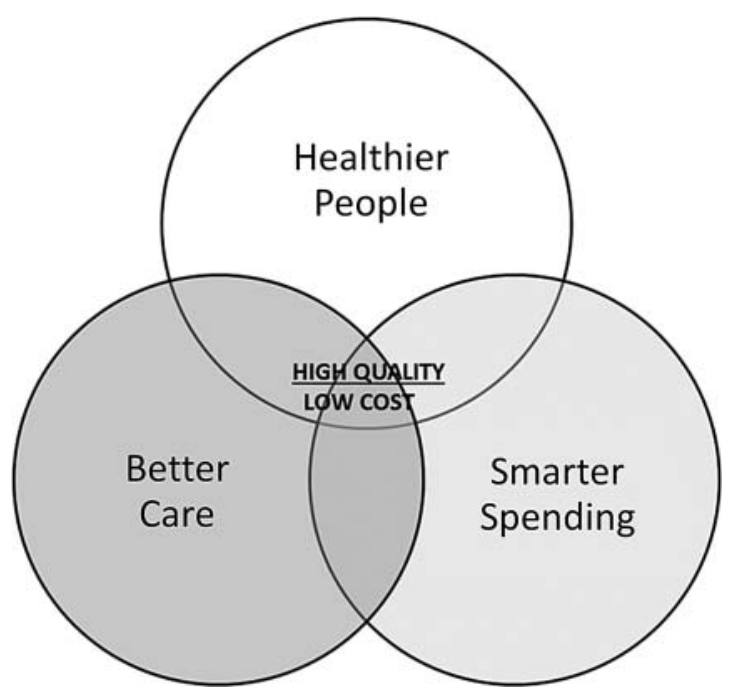

Figure 1. The Institute for Healthcare Improvement Triple Aim model to achieve high-value healthcare. from each hospital department; members of the hospital PI department; the chief quality officer; the chief medical officers; the chief nursing officer; the designated institutional official for GME; and representatives from nursing, infection control, and risk management. In accordance with the CLER, the HSQC engages residents with their peers and hospital administrators with the goal of making alignment between house staff and the hospital a reality rather than just a concept.

To further align house staff priorities with hospital priorities, we mapped house staff PI projects to the UHC domains of care and the IHI Triple Aim. We were then able to perform a gap analysis that led to opportunities to be proactive about alignment when the house staff propose projects.

\section{METHODS}

The HSQC, composed of 17 house staff and 20 faculty and administrative members, undertook this project. The HSQC was created in 2012 to promote resident education in quality and $\mathrm{PI}$ in accordance with Accreditation Council for Graduate Medical Education and CLER recommendations to improve historically low resident participation in $\mathrm{PI}$ initiatives. ${ }^{6}$ Quality councils have been shown to increase involvement of front-line providers in $\mathrm{PI}$ initiatives, which helps enact change at the hospital level. ${ }^{7}$

For a 2-year period, the HSQC collected data on PI projects being done by the house staff in all hospital departments. The majority of the data was collected via survey asking house staff to submit information about their projects. In addition, program directors and chairs independently submitted lists of PI projects being worked on in their departments. The data were organized by department and title to eliminate duplications and to create a comprehensive list.

Once the inventory was completed, a multidisciplinary team of quality experts, consisting of the resident chair of the HSQC, the resident co-chair of the HSQC, the chief quality officer, the director of performance improvement and clinical value, and the perioperative performance improvement manager, was assembled to categorize each house staff quality project according to UHC domain (Table). Each team member categorized each project according to the UHC domain definitions and criteria. The UHC domain of Safety included all iatrogenic infections or injuries and postoperative complications, including those from anesthesia. The Mortality domain included severe illnesses contributing to the death of patients, including myocardial infarction, stroke, gastrointestinal hemorrhage, pneumonia, and hip fracture. The Effectiveness domain focused primarily on readmissions and core measures. The Equity domain focused on the influence of sex, socioeconomic status, and race on all aspects of health. ${ }^{8}$ The Efficiency domain centered around timeliness and cost of care. Finally, the Patient Centeredness domain concentrated on patient satisfaction with pain management and provider communication. ${ }^{9}$

Once the individual members completed their categorizations, the chair of the HSQC assigned a primary domain to each project based on the consensus of the quality team. The projects were then grouped by domain and sorted by specialty to reflect the contributions of the house staff in each department. Once all projects were grouped by domain and department, they were further categorized within the elements of the IHI Triple Aim. 
Because this project did not involve human subject research as defined by the US Department of Health and Human Services or US Food and Drug Administration regulations, the institution's institutional review board (IRB) determined that IRB review and approval were not necessary.

\section{RESULTS}

A total of 184 projects representing 24 departments were identified. The departments with the most PI projects were internal medicine (42), emergency medicine (27), and obstetrics and gynecology (17). Departments with the fewest projects were neurology (1), podiatry (1), and physical medicine and rehabilitation (1). The majority of the projects (38\%), representing 18 departments, were categorized in the Safety domain. The remainder of the projects were classified as follows: $23 \%$ in the Efficiency domain, $12 \%$ in the Patient Centeredness domain, $12 \%$ in the Effectiveness domain, $8 \%$ in the Equity domain, and $7 \%$ in the Mortality domain, representing $16,10,10,8$, and 6 departments, respectively. In absolute comparison, the departments best represented in each UHC domain were as follows: Effectiveness, pathology and internal medicine; Efficiency, internal medicine; Equity, internal medicine; Mortality, emergency medicine; Patient Centeredness, internal medicine; and Safety, internal medicine (Figure 2).

Many departments did not have projects in all domains. In fact, only internal medicine had projects that spanned all 6 UHC domains. Three departments-emergency medicine, general surgery, and obstetrics and gynecology- had projects in 5 of the 6 domains. Only 6 departmentsemergency medicine, gastroenterology, general surgery, internal medicine, radiation oncology, and surgical oncology-had projects in the Mortality domain. Similarly, projects categorized in the Equity domain came only from anesthesia, cardiology, general surgery, hematology/oncology, internal medicine, obstetrics and gynecology, surgical oncology, and urology. The Effectiveness domain included projects from anesthesia, cardiology, emergency medicine, general surgery, infectious disease, internal medicine, obstetrics and gynecology, orthopedic surgery, pathology, and rheumatology.

No projects from anesthesia, cardiology, diagnostic radiology, endocrinology, hematology/oncology, infectious disease, neurology, ophthalmology, pathology, physical medicine and rehabilitation, podiatry, radiation oncology, rheumatology, and urology were included in the Patient Centeredness domain. Likewise, no projects from cardiology, neurology, physical medicine and rehabilitation, rheumatology, surgical oncology, and urology were categorized under the Safety domain. Geriatrics, general surgery, hematology/oncology, infectious disease, ophthalmology, podiatry, psychiatry, and radiation oncology had no projects categorized in the Efficiency domain.

In terms of the IHI Triple Aim, the majority of projects included in the Safety domain spanned the largest portion of all 3 goals of smarter spending, healthier people, and better care (Figure 3). Projects in the Efficiency and Effectiveness domains also spanned all 3 goals, whereas those

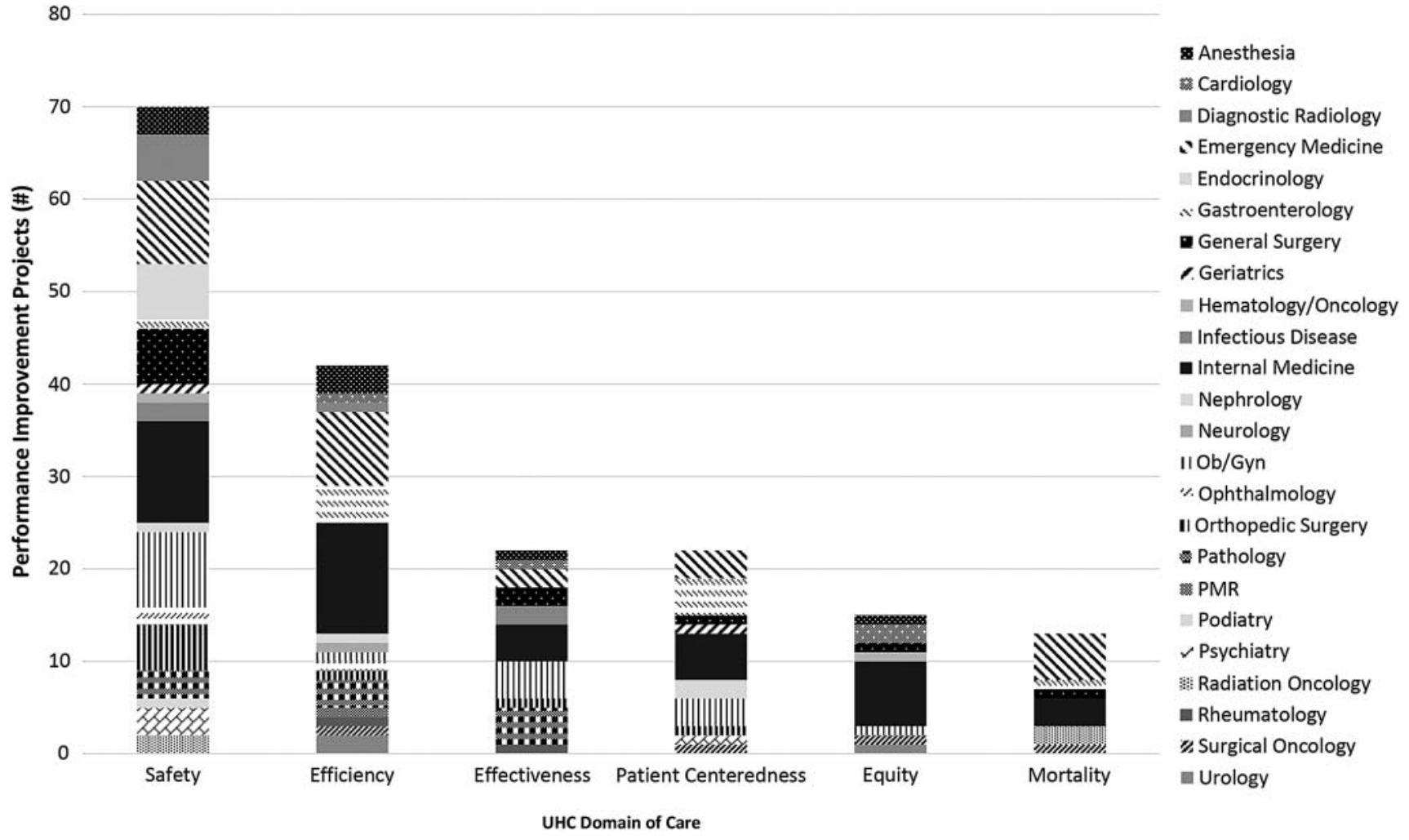

Figure 2. Alignment of house staff performance improvement projects with institutional patient safety and performance improvement priorities: the University HealthSystem Consortium domains of care. Ob/Gyn, obstetrics and gynecology; PMR, physical medicine and rehabilitation. 


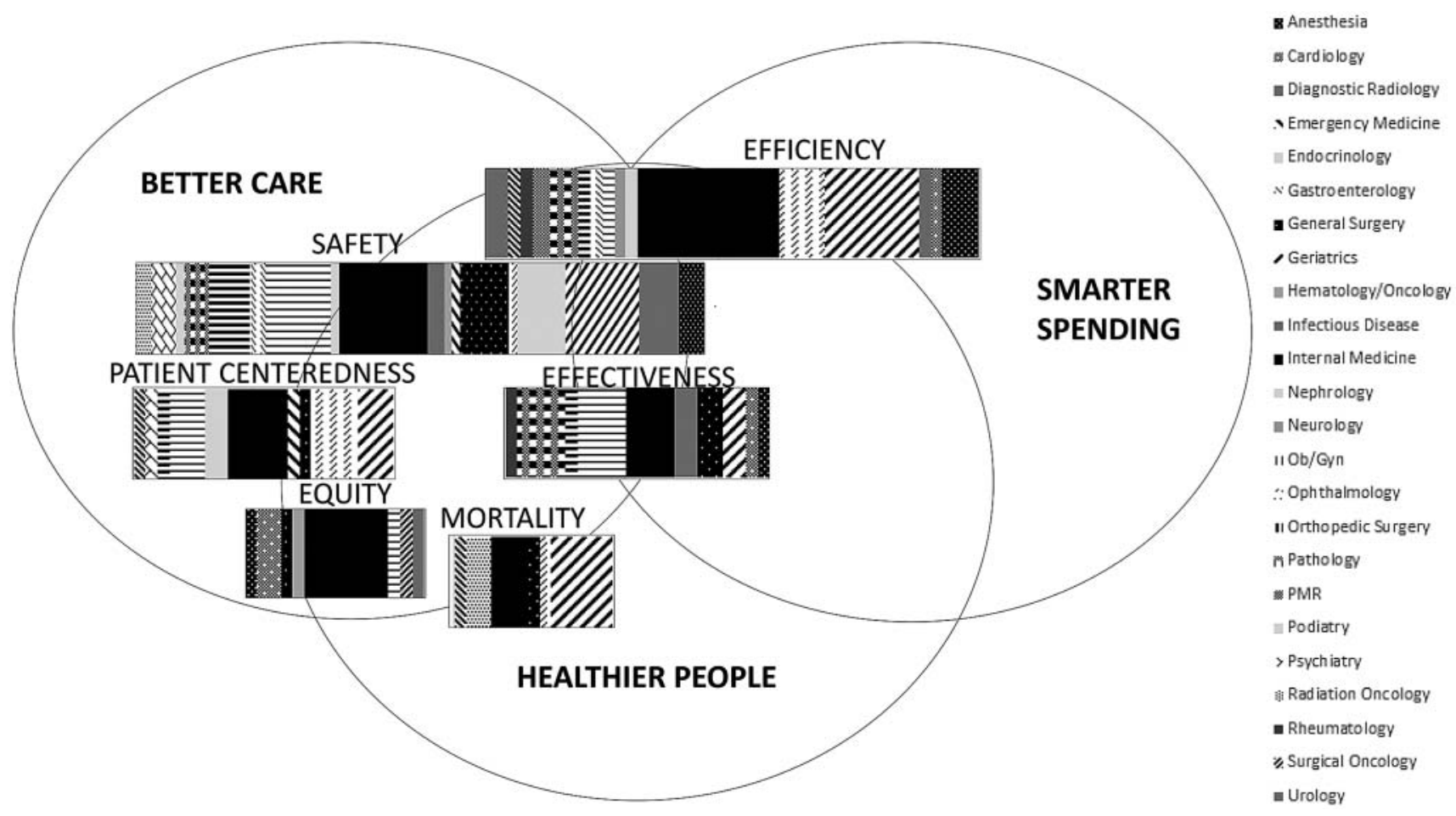

Figure 3. Alignment of house staff performance improvement projects with institutional patient safety and performance improvement priorities: the Institute for Healthcare Improvement Triple Aim. Ob/Gyn, obstetrics and gynecology; PMR, physical medicine and rehabilitation.

included in Equity, Mortality, and Patient Centeredness spanned only 2 goals.

Although the majority of projects included in the data analysis were the work of individual residents or fellows, some projects were the cumulative work of multiple residents. Of the total complement of 553 house staff, 237 (43\%) different participants were involved in the 184 projects included in this study. Thirty-two projects involved multiple house staff. All 10 projects in the pathology department involved multiple residents, as did the one project from podiatry. The departments with the most house staff working on PI projects were internal medicine (95), pathology (35), and emergency medicine (19). The number of participants often exceeded the number of GME-designated slots for house staff in the different departments because the data collection occurred during a 2-year period, and residents or fellows who had already graduated were included in the analysis.

Twenty-nine projects were more focused on care in the outpatient setting than the inpatient setting and therefore may have been more appropriately categorized directly in the IHI Triple Aim rather than the UHC domains of care. ${ }^{5} \mathrm{~A}$ subanalysis of these projects showed that 19 projects were focused on healthier people and 10 projects were focused on better care. No projects focused solely on smarter spending (Figure 4).

\section{DISCUSSION}

This project led to the creation of unique and concise graphic representations demonstrating how individual house staff projects fit into hospitalwide initiatives. Mapping house staff PI projects to hospital priorities created the op- portunity to engage house staff, program directors, faculty, and hospital administration in an effort to align the PI goals of all constituents. The gap analysis suggested that the projects were skewed toward the Safety and Efficiency domains, consistent with recent hospital initiatives to decrease the incidence of hospital-acquired conditions and length of stay and reflecting the house staff focus at the departmental level. Many projects in the Safety domain dealt with postoperative outcomes, procedural complications, or departmental protocol development, all of which are issues the house staff deal with on a daily basis. Likewise, projects that were categorized in the Efficiency domain, principally performed by internal medicine and emergency medicine house staff, focused on throughput and length of stay, issues that affect both departments on a daily basis in a high-volume academic center. These projects relate most closely to the smarter spending element of the IHI Triple Aim and reflect the emphasis on cost containment from the Centers for Medicare and Medicaid Services.

Surprisingly, at a hospital that serves a primarily low socioeconomic demographic, only $8 \%$ of projects were categorized in the Equity domain that is concerned with the effects of race, sex, and socioeconomic class on health outcomes. No clear reason for the lack of projects in this domain was identified. The low number of projects in the Mortality domain was unexpected. One possibility is that residents and fellows focus on mortality in other ways during their training, such as through morbidity and mortality conferences. A hospital mortality review process has been implemented that may provide opportunities for $\mathrm{PI}$ and research not previously appreciated. 


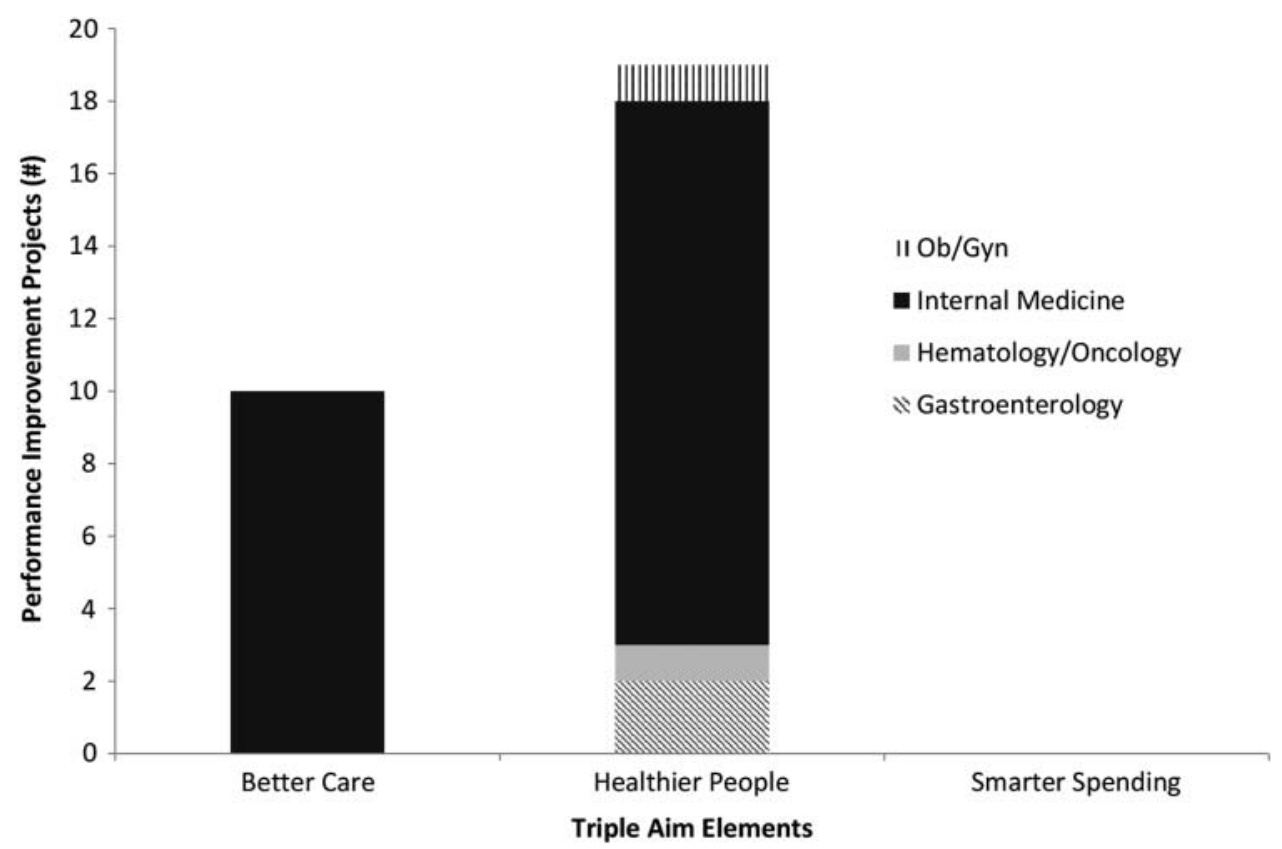

Figure 4. Alignment of house staff outpatient performance improvement projects with institutional patient safety and performance improvement priorities: the Institute for Healthcare Improvement Triple Aim. Ob/Gyn, obstetrics and gynecology.

We now anticipate taking a proactive approach to project alignment; the house staff will identify a best-fit UHC domain and IHI Triple Aim element or elements for their project when they submit it for inclusion in the catalog. This requirement, in turn, will necessitate a concurrent effort to continually educate house staff on the UHC domains and the IHI Triple Aim. We plan to provide this education through brief lectures given by house staff members of the HSQC to their respective departments and by distributing pocket-sized quality cards to all house staff to serve as a quick reference. As more projects are submitted and the project database grows, the gap analysis will be repeated and again shared with house staff and hospital administrators to promote alignment and engagement.

This study had several limitations. The inventory of house staff quality projects was incomplete. All data were subject to response bias, as the only way to accumulate data was to survey house staff and ask program directors and chairs for submissions. Further, the data were collected during a 2year span. Although all projects were active at some point during the 2-year period, some projects may have concluded. Additionally, categorization of some projects in UHC domains was limited by the information provided by house staff in their survey responses.

\section{CONCLUSION}

We have described an innovative way to align individual house staff quality projects with hospital goals and national healthcare agendas in a concise, graphic manner. The subsequent gap analysis identified deficiencies that served as a guide for a proactive approach to align future house staff PI projects with broader healthcare initiatives and hospital priorities. Overall, we created a framework that turned the conceptual alignment of house staff and hospital priorities into a reality.

\section{ACKNOWLEDGMENTS}

The authors have no financial or proprietary interest in the subject matter of this article.

This project was a poster presentation at the Hospital and Healthsystem Association of Pennsylvania Quality and Patient Safety Symposium, September 7-8, 2016.

\section{REFERENCES}

1. Fleischut PM, Faggiani SL, Evans AS, et al. 2011 John M. Eisenberg Patient Safety and Quality Awards. The effect of a novel Housestaff Quality Council on quality and patient safety. Innovation in patient safety and quality at the local level. $J t$ Comm J Qual Patient Saf. 2012 Jul;38(7):311-317.

2. Klein S. Case study: the housestaff quality council at New YorkPresbyterian Hospital/Weill Cornell Medical Center. Quality Matters Archive. http://www.commonwealthfund.org /publications/newsletters/quality-matters/2010/october -november-2010/case-study. Published October/November 2010. Accessed April 2, 2018.

3. Institute of Medicine (US) Committee on Quality of Health Care in America. Crossing the Quality Chasm: A New Health System for the 21st Century. Washington, DC: National Academies Press; 2001.

4. Institute of Medicine (US) Committee on Quality of Health Care in America; Kohn LT, Corrigan JM, Donaldson MS, eds. To Err is Human: Building a Safer Health System. Washington, DC: National Academies Press; 2000.

5. Institute for Healthcare Improvement. IHI Triple Aim Initiative. http://www.ihi.org/engage/initiatives/tripleaim/pages/default .aspx. Accessed June 11, 2016.

6. Dixon JL, Papaconstantinou HT, Erwin JP 3rd, McAllister RK, Berry $\mathrm{T}$, Wehbe-Janek $\mathrm{H}$. House staff quality council: one institution's experience to integrate resident involvement in patient care improvement initiatives. Ochsner J. 2013 Fall;13 (3):394-399. 
7. Stueven J, Sklar DP, Kaloostian $P$, et al. A resident-led institutional patient safety and quality improvement process. Am J Med Qual. 2012 Sep-Oct;27(5):369-376. doi: 10.1177/ 1062860611429387.

8. Keroack MA, Youngberg BJ, Cerese JL, Krsek C, Prellwitz LW, Trevelyan EW. Organizational factors associated with high performance in quality and safety in academic medical centers. Acad Med. 2007 Dec;82(12):1178-1186.
9. University HealthSystem Consortium. 2010 UHC Quality and Safety Fall Forum. UHC 2010 Quality and Accountability Study: Scoring and Ranking Methodology. https://healthcare.utah.edu /publicaffairs/pdfs/uhc-2010.pdf. Accessed April 2, 2018.

This article meets the Accreditation Council for Graduate Medical Education and the American Board of Medical Specialties Maintenance of Certification competencies for Patient Care, Medical Knowledge, and Systems-Based Practice. 